\title{
PROJECTED THREE-POINT CORRELATION FUNCTIONS AND GALAXY BIAS
}

\author{
ZHENG ZHENG \\ Department of Astronomy, Ohio State University, Columbus, OH 43210; zhengz@astronomy.ohio-state.edu \\ Received 2004 May 26; accepted 2004 June 29
}

\begin{abstract}
The three-point correlation function (3PCF) can now be measured in large galaxy redshift surveys, but in three dimensions its interpretation is complicated by the presence of redshift-space distortions. I investigate the projected 3PCF, where these distortions are eliminated by integrating over the redshift dimension, as is commonly done for the two-point correlation function. The calculation of the projected 3PCF from the real-space, threedimensional bispectrum is greatly simplified by expanding both quantities in Fourier components, analogous to Szapudi's expansion of the three-dimensional quantities in multipole components. In the weakly nonlinear regime, the bispectrum can be well represented by the first few Fourier components. There is a well-known relation between the reduced 3PCFs of matter and galaxies in the weakly nonlinear regime, which can be used to infer galaxy bias factors if the real-space three-dimensional galaxy correlation functions (two-point and three-point) can be measured. I show that the same relation holds for the reduced projected 3PCFs if these are properly defined. These results should aid determinations of galaxy bias from large redshift surveys by eliminating the complication of redshift-space distortions.
\end{abstract}

Subject headings: cosmology: theory — dark matter — galaxies: formation — galaxies: halos — galaxies: statistics - large-scale structure of universe

\section{INTRODUCTION}

The three-point correlation function (3PCF), or its Fourier transform, the bispectrum, is a valuable complement to twopoint statistics in characterizing galaxy clustering. The behavior of the 3PCF of the matter is well understood in perturbation theory (e.g., Fry 1984; Goroff et al. 1986; Bernardeau 1992; Jain \& Bertschinger 1994; Scoccimarro \& Frieman 1996, 1999; Scoccimarro et al. 1998; Bernardeau et al. 2002). For Gaussian initial conditions, second-order perturbation theory predicts that the amplitude of the 3PCF $\xi^{[3]}$ scales like the square of the amplitude of the two-point correlation function (2PCF) $\xi$ (Peebles 1980), and this scaling is one of the fundamental tests of the Gaussian primordial fluctuations predicted by inflationary cosmology. Nonlinear gravitational evolution produces anisotropic, filamentary structures, so elongated triangle configurations have higher amplitude in the three-point statistics. Galaxy bias, a difference between the distributions of galaxies and matter, can alter three-point statistics, but it tends to boost or suppress amplitudes for all triangle configurations equally, at least on large scales. The triangle shape dependence of the 3PCF or bispectrum, in combination with the 2PCF or power spectrum, therefore becomes a diagnostic for galaxy bias and the matter clustering amplitude (Fry 1994).

Early measurements of the 3PCF or bispectrum were based on angular catalogs (e.g., Peebles \& Groth 1975; Fry \& Peebles 1980; Jing et al. 1991; Fry 1994). Galaxy redshift surveys like the Two-Degree Field Galaxy Redshift Survey (2dFGRS; Colless et al. 2001) and the Sloan Digital Sky Survey (SDSS; York et al. 2000) are now large enough to allow measurements of the redshift-space 3PCF or bispectrum with high signal-tonoise ratio (e.g., Verde et al. 2002; Jing \& Börner 2004; Kayo et al. 2004). However, this brings in the additional complication of distortion by the peculiar motions of galaxies. Even for the 2PCF or power spectrum, the effect of nonlinear redshift-space distortions persist to remarkably large scales (Cole et al. 1994). Distortions of the 3PCF or bispectrum are more complex, and while some models of these distortions exist (e.g., Scoccimarro et al. 2001; Verde et al. 2002), it is not clear that they are accurate at the level of precision afforded by current data. An obvious way to circumvent redshift-space distortions is to project the 3PCF over the redshift direction, as is commonly done for the projected 2PCF $w_{p}\left(r_{p}\right)$ (Davis \& Peebles 1983). We note that projection of redshift surveys is not the same as simply measuring from the parent angular catalog - the use of galaxy redshifts to obtain physical projected separations greatly reduces the noise and yields a quantity more closely related to the three-dimensional 3PCF. Projected 3PCFs have been measured for the Las Campanas Redshift Survey (LCRS) by Jing \& Börner (1998) and for the 2dFGRS (Jing \& Börner 2004) on relatively small scales. By comparing the measurements with the predicted 3PCFs of matter in $N$-body simulations, they find that the observed galaxy 3PCFs are lower than the predicted matter 3PCFs, indicating the need of galaxy bias to explain the data.

Since the three-dimensional 3PCF is already a complicated object, projection may seem to forgo any chance of analytic treatment. However, I show here that the relation of the projected 3PCF to the (three-dimensional) bispectrum is relatively straightforward if the bispectrum is decomposed into Fourier moments $(\S 2)$. My analysis is directly analogous to that of Szapudi (2004), who introduces multipole expansion of the three-dimensional 3PCF and bispectrum and shows it to be useful in simplifying the relation between them, in characterizing the triangle configuration dependence, and in constraining galaxy bias. The spirit of this paper parallels that of Szapudi (2004). In $\S 3$ I show that bias effects remain simple for projected 2PCFs and 3PCFs on large scales, which makes it attractive to constrain galaxy bias using projected quantities. I briefly summarize my results in $\S 4$.

\section{FOURIER EXPANSION AND PROJECTED 3PCFs}

Before going into details, I emphasize that the meaning of projection in this paper has subtle differences from the usual 
angular correlation. Our goal is to describe the projection of the measured redshift-space correlation functions from a galaxy redshift survey and to use it to study problems like galaxy bias. Since galaxies with different properties cluster differently (e.g., red galaxies are in general more strongly clustered than blue galaxies), it is desirable to construct volume-limited samples that uniformly represent galaxies of a given type. By doing so, we can infer bias information as a function of galaxy type, rather than some average over all types of galaxies (weighted in a complicated way) like that from flux-limited galaxy samples. This approach helps the understanding of galaxy bias and aids comparisons with theoretical structure formation models. Redshiftspace correlation functions can be measured by comparing the galaxy distribution with a distribution of random points that has the same geometry and selection function as the galaxies (e.g., Landy \& Szalay 1993). The projected correlation function can be formed by integrating the redshift-space correlation function along the redshift direction. The selection function does not enter the integration because its effect is included in the error budget of the measured redshift-space correlation function. In contrast, one needs to take into account the selection function in modeling angular correlation measurements of galaxy clustering. Nevertheless, our results in this paper can be easily generalized to include selection function to model angular clustering (see the discussion in $\S 3$ and Fry \& Thomas 1999).

Since redshift-space distortions conserve numbers of pairs and triplets, 2PCFs and 3PCFs projected from real-space threedimensional correlation functions are identical to those from redshift-space three-dimensional correlation functions. This vastly simplifies theoretical predictions of projected 2PCFs and $3 \mathrm{PCF}$ - we only need to project the real-space threedimensional correlation functions, without calculating redshiftspace distortions. In practice, the radial extent of the projection may not be infinite, but it should be large enough to minimize any residual redshift-space distortions. We generally assume ideal infinite projections and plane-parallel geometry (the distant observer approximation) in our derivation.

\subsection{Projected $2 \mathrm{PCFS}$}

We start by reviewing the calculation of the projected $2 \mathrm{PCF}$ and its relation to the fluctuation power spectrum. The (threedimensional) 2PCF $\xi(\boldsymbol{r})$ is the Fourier transform of the fluctuation power spectrum $P(\boldsymbol{k})$,

$$
\xi(\boldsymbol{r})=\frac{1}{(2 \pi)^{3}} \int d^{3} \boldsymbol{k} P(\boldsymbol{k}) e^{i \boldsymbol{k} \cdot \boldsymbol{r}},
$$

where $\boldsymbol{r}$ is the pair separation. Equation (1) holds for real-space quantities, which are isotropic, or for redshift-space quantities, which are not. Both $\boldsymbol{r}$ and $\boldsymbol{k}$ can be decomposed into components perpendicular and parallel to the line of sight: $\boldsymbol{r}=\boldsymbol{r}_{p}+\boldsymbol{r}_{\|}$ and $\boldsymbol{k}=\boldsymbol{k}_{p}+\boldsymbol{k}_{\|}$. The projected 2PCF is obtained by integrating $\xi(\boldsymbol{r})$ along the line of sight,

$$
w_{p}\left(\boldsymbol{r}_{p}\right)=\int_{-\infty}^{+\infty} d r_{\|} \xi\left(\boldsymbol{r}_{p}+\boldsymbol{r}_{\|}\right)
$$

After substituting equation (1) into equation (2) and putting variables into the form of perpendicular and parallel components, we have

$$
w_{p}\left(\boldsymbol{r}_{p}\right)=\frac{1}{(2 \pi)^{3}} \int d^{2} \boldsymbol{k}_{p} e^{i \boldsymbol{k}_{p} \cdot \boldsymbol{r}_{p}} \int_{-\infty}^{+\infty} d k_{\|} P\left(\boldsymbol{k}_{p}+\boldsymbol{k}_{\|}\right) \int_{-\infty}^{+\infty} d r_{\|} e^{i k_{\|} r_{\|}}
$$

$$
=\frac{1}{(2 \pi)^{2}} \int d^{2} \boldsymbol{k}_{p} P\left(\boldsymbol{k}_{p}\right) e^{i \boldsymbol{k}_{p} \cdot \boldsymbol{r}_{p}}
$$

Equation (4) follows from equation (3) because the rightmost integral in equation (3) is just $2 \pi$ times the Dirac $\delta$-function $\delta_{D}\left(k_{\|}\right)$. Equation (4) states that the projected 2PCF is the two-dimensional Fourier transform of the power spectrum, and because we have projected out redshift-space distortions, we can use the isotropic, real-space $P(k)$ in the integral. We can evaluate equation (4) in polar coordinates. The angular part can be calculated by using the expansion $\exp (i \boldsymbol{k} \cdot \boldsymbol{r})=$ $\sum_{n=-\infty}^{+\infty} J_{n}(k r) i^{n} \exp [i n(\phi-\Phi)]$ (plane waves in terms of cylindrical waves), where $\phi-\Phi$ is the angle between $\boldsymbol{k}$ and $\boldsymbol{r}$ and $J_{n}(x)$ is the Bessel function of integer order. Finally, equation (4) reduces to a one-dimensional integral,

$$
w_{p}\left(r_{p}\right)=\int_{0}^{\infty} \frac{k}{2 \pi} d k P(k) J_{0}\left(k r_{p}\right) .
$$

This equation mimics the relation for the real-space, threedimensional 2PCF, which involves the spherical Bessel function $j_{0}(x)$. This kind of result can be found in papers that deal with projected observations, such as variants of Limber's equation (e.g., Baugh \& Efstathiou 1993).

\subsection{Projected $3 P C F S$}

The projected 3PCFs can be derived in a similar way to the 2PCFs. The three-dimensional 3PCF is the Fourier transform of the bispectrum $B\left(\boldsymbol{k}_{1}, \boldsymbol{k}_{2}, \boldsymbol{k}_{3}\right)$,

$$
\begin{aligned}
\xi^{[3]}\left(\boldsymbol{r}_{1}, \boldsymbol{r}_{2}, \boldsymbol{r}_{3}\right)= & \frac{1}{(2 \pi)^{6}} \int d^{3} \boldsymbol{k}_{1} d^{3} \boldsymbol{k}_{2} d^{3} \boldsymbol{k}_{3} B\left(\boldsymbol{k}_{1}, \boldsymbol{k}_{2}, \boldsymbol{k}_{3}\right) \\
& \times e^{i\left(\boldsymbol{k}_{1} \cdot \boldsymbol{r}_{1}+\boldsymbol{k}_{2} \cdot \boldsymbol{r}_{2}+\boldsymbol{k}_{3} \cdot \boldsymbol{r}_{3}\right)} \delta_{D}\left(\boldsymbol{k}_{1}+\boldsymbol{k}_{2}+\boldsymbol{k}_{3}\right) .
\end{aligned}
$$

The Dirac $\delta$-function selects wavevectors $\left(\boldsymbol{k}_{1}, \boldsymbol{k}_{2}, \boldsymbol{k}_{3}\right)$ that form a triangle. From now on, we adopt the notation that a subscript of one number represents one side for a wavevector triangle, while it represents one vertex for a real-space triangle. Each side of a real-space triangle is denoted by subscript of two numbers, e.g., $\boldsymbol{r}_{i j}=\boldsymbol{r}_{i}-\boldsymbol{r}_{j}(i, j=1,2,3)$. Equation (6) reduces to

$$
\begin{aligned}
\xi^{[3]}\left(\boldsymbol{r}_{1}, \boldsymbol{r}_{2}, \boldsymbol{r}_{3}\right)= & \frac{1}{(2 \pi)^{6}} \int d^{3} \boldsymbol{k}_{1} d^{3} \boldsymbol{k}_{2} B\left(\boldsymbol{k}_{1}, \boldsymbol{k}_{2}\right) \\
& \times e^{i\left(\boldsymbol{k}_{1} \cdot \boldsymbol{r}_{13}+\boldsymbol{k}_{2} \cdot \boldsymbol{r}_{23}\right)},
\end{aligned}
$$

where $\boldsymbol{k}_{3}$ does not appear explicitly in the bispectrum because the wavevector triangle is fully determined by $\boldsymbol{k}_{1}$ and $\boldsymbol{k}_{2}\left(\boldsymbol{k}_{1}+\right.$ $\left.\boldsymbol{k}_{2}+\boldsymbol{k}_{3}=0\right)$.

Similar to the projected $2 \mathrm{PCF}$, the projected 3PCF for a projected triangle with $\left(\boldsymbol{r}_{p 1}, \boldsymbol{r}_{p 2}, \boldsymbol{r}_{p 3}\right)$ as vertices can be obtained by integrating $\xi^{[3]}$ along the line of sight. One vertex (e.g., $\boldsymbol{r}_{p 3}$ ) can be fixed. We then have the following expression for the projected $3 \mathrm{PCF}$,

$$
\begin{aligned}
w_{p}^{[3]}\left(\boldsymbol{r}_{p 1}, \boldsymbol{r}_{p 2}, \boldsymbol{r}_{p 3}\right)= & \int_{-\infty}^{+\infty} d r_{\| 1} \int_{-\infty}^{+\infty} d r_{\| 2} \\
& \times \xi^{[3]}\left(\boldsymbol{r}_{p 1}+\boldsymbol{r}_{\| 1}, \boldsymbol{r}_{p 2}+\boldsymbol{r}_{\| 2}, \boldsymbol{r}_{p 3}\right) .
\end{aligned}
$$

Substituting equation (7) into the above, changing to cylindrical coordinates, and following the procedures used to go from 
equation (3) to equation (4), we can derive the relation between the projected $3 \mathrm{PCF}$ and the bispectrum

$$
\begin{aligned}
w_{p}^{[3]}\left(\boldsymbol{r}_{p 1}, \boldsymbol{r}_{p 2}, \boldsymbol{r}_{p 3}\right)= & \frac{1}{(2 \pi)^{4}} \int d^{2} \boldsymbol{k}_{p 1} d^{2} \boldsymbol{k}_{p 2} B\left(\boldsymbol{k}_{p 1}, \boldsymbol{k}_{p 2}\right) \\
& \times e^{i \boldsymbol{k}_{p 1} \cdot \boldsymbol{r}_{p 13}} e^{i \boldsymbol{k}_{p 2} \cdot \boldsymbol{r}_{p 23}} .
\end{aligned}
$$

That is, the projected 3PCF is the two-dimensional Fourier transform of the bispectrum. Equation (9) is a four-dimensional integral, and it is clearly desirable to simplify the calculation, by reducing its dimensionality. Note that $B\left(\boldsymbol{k}_{p 1}, \boldsymbol{k}_{p 2}\right)$ is just the usual three-dimensional, real-space bispectrum evaluated at $\boldsymbol{k}_{1}=\boldsymbol{k}_{p 1}, \boldsymbol{k}_{2}=\boldsymbol{k}_{p 2}$, and $\boldsymbol{k}_{3}=-\left(\boldsymbol{k}_{p 1}+\boldsymbol{k}_{p 2}\right)$.

Szapudi (2004) introduces multipole expansion of the threedimensional three-point statistics and shows that the 3PCF can then be put in a simple form (see also Verde et al. 2000 for a similar expansion in the bispectrum). Consequently, the whole calculation becomes simple because only a few multipoles are needed for accurate convergence. In the same spirit as Szapudi (2004), we introduce Fourier expansion of the projected threepoint statistics. The bispectrum can be expanded as

$$
B\left(k_{1}, k_{2}, \phi\right)=\sum_{n=-\infty}^{+\infty} B_{n}\left(k_{1}, k_{2}\right) e^{i n \phi},
$$

where $\phi$ is the angle between $\boldsymbol{k}_{1}$ and $\boldsymbol{k}_{2}$, and the coefficient $B_{n}\left(k_{1}, k_{2}\right)$ can be obtained through

$$
\begin{aligned}
B_{n}\left(k_{1}, k_{2}\right) & =\frac{1}{2 \pi} \int_{0}^{2 \pi} d \phi B\left(k_{1}, k_{2}, \phi\right) e^{i n \phi} \\
& =\frac{1}{2 \pi} \int_{0}^{2 \pi} d \phi B\left(k_{1}, k_{2}, \phi\right) \cos n \phi .
\end{aligned}
$$

We have used the fact that the bispectrum has the symmetry $B\left(k_{1}, k_{2},-\phi\right)=B\left(k_{1}, k_{2}, \phi\right)$, so $B_{-n}=B_{n}$ and the expansion is a cosine Fourier expansion.

We rewrite equation (9) in polar variables, replace $B$ with its Fourier expansion, and expand the two exponentials in the same way as we do in deriving equation (5) (it is useful to write the angle between two vectors as the difference of their polar angles). After integrating the angular part using the orthogonality of $\exp (i n \phi)$, we find that

$$
w_{p}^{[3]}\left(r_{p 13}, r_{p 23}, \Phi\right)=\sum_{n=-\infty}^{+\infty} w_{p, n}^{[3]}\left(r_{p 13}, r_{p 23}\right) e^{i n \Phi},
$$

where $\Phi$ is the angle between $\boldsymbol{r}_{p 13}$ and $\boldsymbol{r}_{p 23}$, and the Fourier coefficient

$$
\begin{aligned}
w_{p, n}^{[3]}\left(r_{p 13}, r_{p 23}\right)= & \int_{0}^{\infty} \frac{k_{1}}{2 \pi} d k_{1} \int_{0}^{\infty} \frac{k_{2}}{2 \pi} d k_{2} \\
& \times(-1)^{n} B_{n}\left(k_{1}, k_{2}\right) J_{n}\left(k_{1} r_{p 13}\right) J_{n}\left(k_{2} r_{p 23}\right) .
\end{aligned}
$$

Equation (13) is in a form that resembles the projected 2PCF in equation (5). The essence here is that, by Fourier expansion of the bispectrum $B$ and cylindrical wave expansion of the plane wave $\exp \left(\boldsymbol{i}_{p} \cdot \boldsymbol{r}_{p}\right)$, the angular dependence is separated and integrated. There are also similarities between the above equation and equation (3) in Szapudi (2004) for the multipole coefficient of the three-dimensional 3PCF. We see that the calculation of the projected 3PCF from a given three-dimensional bispectrum requires essentially the same amount of work as the three-dimensional 3PCF (and a similar remark holds for 2PCF and the three-dimensional power spectrum). For 3PCFs, what we need to calculate are expansion coefficients, where only onedimensional (for expansion coefficients of the bispectrum) and two-dimensional (for coefficients of the correlation function) integrals are involved. I note that the same result (eq. [13]) is also obtained independently by I. Szapudi (2004, private communication).

If the effect of finite projection has to be taken into account, the Dirac $\delta$-function we use to derive equations (4) and (9) is replaced by $r_{\|, \max } j_{0}\left(k_{\|} r_{\|, \max }\right) / \pi$, if the projection is performed for line-of-sight separations from $-r_{\|, \max }$ to $r_{\|, \max }$. Equations (4) and (9) become three- and six-dimensional integrals, respectively. The Fourier expansion can still be adopted to reduce the calculation of the projected 3PCF to four-dimensional integrals.

As an example of the advantage of the Fourier expansion, Figure 1 shows the first few Fourier expansion coefficients of the matter bispectrum for $k_{1}=2 k_{2}=0.05 h \mathrm{Mpc}^{-1}$ and the bispectrum recovered from using Fourier components up to $n=3,5$, and 10 in the weakly nonlinear regime. The matter bispectrum in this regime can be written in the following form (e.g., Fry 1984),

$$
\begin{aligned}
B\left(k_{1}, k_{2}, \phi\right)= & {\left[\left(\frac{3}{2}-\frac{1}{2} \mu\right)+\left(\frac{k_{1}}{k_{2}}+\frac{k_{2}}{k_{1}}\right) \cos \phi\right.} \\
& \left.+\frac{1}{2}(1-\mu) \cos 2 \phi\right] \times P\left(k_{1}\right) P\left(k_{2}\right)+\text { perm. }
\end{aligned}
$$

where $P(k)$ is the linear matter power spectrum and $\mu$ reflects the weak dependence on cosmology $\left(\mu=3 \Omega_{m}^{-1 / 140} / 7 \approx 3 / 7\right.$ for a spatially flat universe; Kamionkowski \& Buchalter 1999; see also Matsubara 1995). The first term has Fourier components only up to $n=2$. The other permutations have higher frequency components because $k_{3}, \boldsymbol{k}_{1} \cdot \boldsymbol{k}_{3}$, and $\boldsymbol{k}_{2} \cdot \boldsymbol{k}_{3}$ are all functions of $\cos \phi$.

The results in Figure 1 are analogous to those of Szapudi (2004), where the bispectrum is viewed as contributions by multipoles. In fact, the $n$ th-order Legendre polynomial has Fourier components up to $n$. In Figure 1 we have adopted a linear power spectrum in the parameterization of Efstathiou et al. (1992) with a primordial fluctuation power-law index $n_{s}=1$ and a shape parameter $\Gamma=0.21$. We see that only a few, low-frequency Fourier components are significant. For example, truncating the Fourier series up to $n=5$ can recover the bispectrum with a fractional error of $\sim 1 \%$ for $k_{1}=2 k_{2}=$ $0.05 h \mathrm{Mpc}^{-1}$. In the weakly nonlinear regime, the galaxy bispectrum can be obtained by a linear combination of the matter bispectrum and the quantity $P\left(k_{1}\right) P\left(k_{2}\right)+P\left(k_{2}\right) P\left(k_{3}\right)+$ $P\left(k_{3}\right) P\left(k_{1}\right)$ with combination coefficients depending on galaxy bias (e.g., Fry 1994). In modeling galaxy 3PCFs, it is useful to expand this quantity in Fourier series, too. Again, the first few Fourier coefficients are enough.

\section{REDUCED PROJECTED 3PCFS AND GALAXY BIAS}

The reduced $3 \mathrm{PCF}$ in real space is defined as

$$
Q\left(\boldsymbol{r}_{1}, \boldsymbol{r}_{2}, \boldsymbol{r}_{3}\right)=\frac{\xi^{[3]}\left(\boldsymbol{r}_{1}, \boldsymbol{r}_{2}, \boldsymbol{r}_{3}\right)}{\xi\left(r_{12}\right) \xi\left(r_{23}\right)+\xi\left(r_{23}\right) \xi\left(r_{31}\right)+\xi\left(r_{31}\right) \xi\left(r_{12}\right)} .
$$



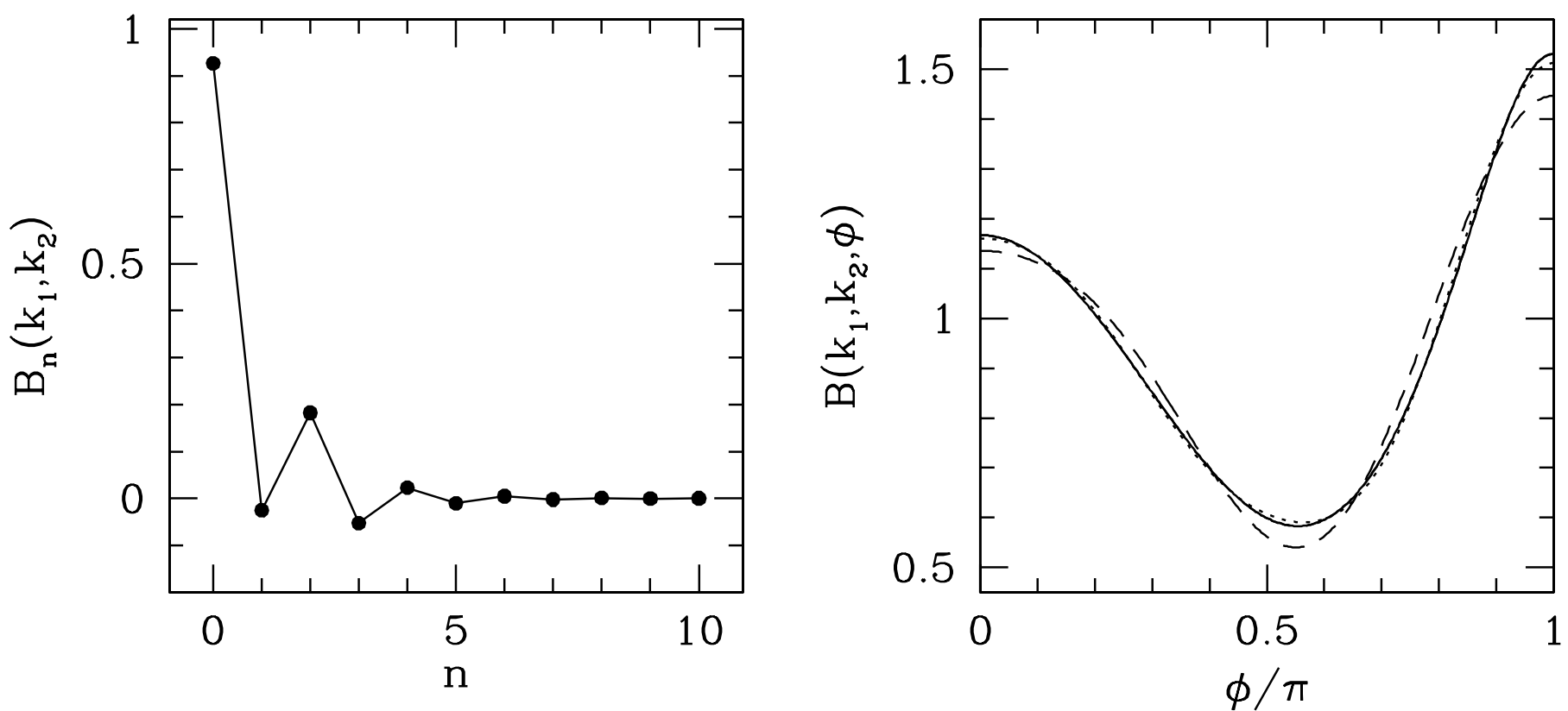

Fig. 1. -Weakly nonlinear matter bispectrum and Fourier expansion coefficients for $k_{1}=2 k_{2}=0.05 h^{-1} \mathrm{Mpc}$. The left panel shows the Fourier coefficients $B_{n}$, which become virtually zero for $n>5$. The right panel shows the bispectrum $B$ (solid curve) as a function of the angle $\phi$ between $\boldsymbol{k}_{1}$ and $\boldsymbol{k}_{2}$ and bispectra reconstructed from the first few Fourier components. The bispectra from the Fourier series up to $n=3$ (dashed curve), 5 (dotted curve), and 10 (dot-dashed curve, indistinguishable from the solid curve) have maximum fractional errors of $\sim 7 \%, \sim 1.5 \%$, and $\sim 0.04 \%$, respectively. A cold dark matter linear power spectrum is assumed (see the text). Both $B$ and $B_{n}$ are normalized by dividing $3 P\left(k_{1}\right) P\left(k_{2}\right)$ for display.

For galaxies, this quantity has contributions from both gravity and galaxy bias (e.g., Fry \& Gaztañaga 1994; Fry 1994; Juszkiewicz et al. 1995). For Gaussian initial conditions, in the weakly nonlinear regime, if the local galaxy overdensity $\delta_{g}$ is expanded in terms of the local matter overdensity $\delta_{m}$ as $\delta_{q}=\sum b_{n} \delta_{m}^{n} / n$ ! (local bias model), the reduced 3PCFs of galaxies and matter have the relation

$$
Q_{g}\left(\boldsymbol{r}_{1}, \boldsymbol{r}_{2}, \boldsymbol{r}_{3}\right)=\frac{1}{b} Q_{m}\left(\boldsymbol{r}_{1}, \boldsymbol{r}_{2}, \boldsymbol{r}_{3}\right)+\frac{b_{2}}{b^{2}},
$$

where $b=b_{1}$ is the linear galaxy bias factor and $b_{2}$ is the lowest-order nonlinear bias factor. In redshift space, the $3 \mathrm{PCF}$ of galaxies, which is easy to measure, differs from the realspace one because of the additional contribution from peculiar motions of galaxies, and the above relation no longer holds. Constraints on galaxy bias from observations in redshift space therefore require accurate modeling of the peculiar motion of galaxies. Since projection along the line of sight essentially eliminates the effect of redshift-space distortions, it is interesting to see whether we can infer $b$ and $b_{2}$ using measurements of projected quantities.

At first glance, it seems impossible to directly perform projections on both sides of equation (16), since the $b_{2} / b^{2}$ term leads to divergence. Furthermore, the projection of $Q$ from redshift space is not, in general, the same as from real space. We therefore project the $2 \mathrm{PCF}$ and $3 \mathrm{PCF}$ s themselves, before taking ratios. To do so, we substitute the definition of $Q$ (eq. [15]) into equation (16) and multiply both sides by $\left[\xi_{g}\left(r_{p 12}\right) \xi_{g}\left(r_{p 23}\right)+\right.$ perm.]. On large scales, by using the relation $\xi_{g}=b^{2} \xi_{m}$, we find

$$
\begin{aligned}
\xi_{g}^{[3]}\left(\boldsymbol{r}_{1}, \boldsymbol{r}_{2},\right. & \left.\boldsymbol{r}_{3}\right)=b^{3} \xi_{m}^{[3]}\left(\boldsymbol{r}_{1}, \boldsymbol{r}_{2}, \boldsymbol{r}_{3}\right) \\
+ & \frac{b_{2}}{b^{2}}\left[\xi_{g}\left(r_{12}\right) \xi_{g}\left(r_{23}\right)+\xi_{g}\left(r_{23}\right) \xi_{g}\left(r_{31}\right)+\xi_{g}\left(r_{31}\right) \xi_{g}\left(r_{12}\right)\right] .
\end{aligned}
$$

Now we can perform projection on both sides by fixing $\boldsymbol{r}_{p 3}$, as we do in equation (8). After the projection, $\xi^{[3]}$ becomes $w_{p}^{[3]}$, and it is easy to show that the product $\xi_{g}\left(r_{i j}\right) \xi_{g}\left(r_{j k}\right)$ becomes $w_{p, g}\left(r_{p i j}\right) w_{p, g}\left(r_{p j k}\right)$. We then divide both sides by $\left[w_{p, g}\left(r_{p 12}\right) w_{p, g}\left(r_{p 23}\right)+\right.$ perm. $]$ and use the linear bias relation $w_{p, g}=b^{2} w_{p, m}$ on large scales. The result is just equation (16) in terms of the reduced projected $3 \mathrm{PCF} Q_{p}$, where $Q_{p}$ is formed as

$$
\begin{aligned}
& Q_{p}\left(\boldsymbol{r}_{p 1}, \boldsymbol{r}_{p 2}, \boldsymbol{r}_{p 3}\right) \\
& =\frac{w_{p}^{[3]}\left(\boldsymbol{r}_{p 1}, \boldsymbol{r}_{p 2}, \boldsymbol{r}_{p 3}\right)}{w_{p}\left(r_{p 12}\right) w_{p}\left(r_{p 23}\right)+w_{p}\left(r_{p 23}\right) w_{p}\left(r_{p 31}\right)+w_{p}\left(r_{p 31}\right) w_{p}\left(r_{p 12}\right)} .
\end{aligned}
$$

So the relation between galaxy and matter reduced 3PCFs in equation (16) still holds,

$$
Q_{p, g}=\frac{1}{b} Q_{p, m}+\frac{b_{2}}{b^{2}},
$$

if the reduced projected 3PCF is properly defined. This kind of definition is also adopted by Jing \& Börner $(1998,2004)$, who point out that if $Q$ is a constant (which in general it is not), then $Q_{p}$ is also a constant and equal to $Q$. Jing \& Börner (1998, 2004 ) also measure the reduced projected $3 \mathrm{PCF}$ as a function of triangle shape for galaxies in the LCRS and the 2dFGRS.

Based on the above results, it is straightforward to infer bias from projected quantities. By projecting the measured redshift-space $\xi$ and $\xi^{[3]}$, we form $Q_{p, q}$ for projected triangles that have two sides $\left(r_{p 13}\right.$ and $\left.r_{p 23}\right)$ fixed but differ in the angle $\Phi$ between them. The dark matter $Q_{p, m}$ can be computed using the method introduced in $\S 2$ (for projecting $\xi$ and $\xi^{[3]}$ ) or can be measured from cosmological $N$-body simulations. The reduced 3PCFs $Q_{p, g}\left(r_{p 13}, r_{p 23}, \Phi\right)$ and $Q_{p, m}\left(r_{p 13}, r_{p 23}, \Phi\right)$ can be expanded in either Fourier or multipole series, whichever one 
prefers. Their expansion coefficients satisfy $Q_{p, g, n}\left(r_{p 13}, r_{p 23}\right)=$ $Q_{p, m, n}\left(r_{p 13}, r_{p 23}\right) / b+\delta_{0 n} b_{2} / b^{2}$, where $\delta_{0 n}=1$ for $n=0$, and 0 otherwise. The nonlinear bias factor only enters in the $n=0$ component (i.e., the DC component for Fourier expansion or the monopole for multipole expansion). The relation between coefficients of other components provide useful tests of bias models and perturbation theory (see $\S 4$ of Szapudi 2004 for a more detailed discussion, which also applies here). Alternatively, we can infer $b$ and $b_{2}$ from a two-parameter fit to $Q_{p, g}\left(r_{p 13}, r_{p 23}, \Phi\right)$, which is widely used (e.g., Fry 1994; Feldman et al. 2001; Scoccimarro et al. 2001).

In order to make meaningful comparisons with predictions in the weakly nonlinear regime, all quantities should be measured on large scales. If the two sides $\left(r_{p 13}\right.$ and $\left.r_{p 23}\right)$ of the projected triangle are large (e.g., greater than $10 h^{-1} \mathrm{Mpc}$ ) and differ substantially (e.g., $\left|r_{p 13}-r_{p 23}\right|>5 h^{-1} \mathrm{Mpc}$ ), then the condition is guaranteed because any deprojected triangle lies in the weakly nonlinear regime (e.g., $r>5 h^{-1} \mathrm{Mpc}$ ). For configurations with $r_{p 13} \approx r_{p 23}$ (but still large), the strongly nonlinear regime affects the value of $Q_{p}$ at $\Phi \sim 0$, where $r_{p 12}$ approaches the size of the largest halos. Even for this extreme case, we can fit $Q_{p, g}$ by rejecting data points near $\Phi=0$. Since the strong nonlinear effect tends to add high-frequency components or higher order multipoles to $Q_{p}$, it is unlikely for it to have a large impact on bias parameters estimated from Fourier coefficients or multipole coefficients. The use of projected triangles with different values of $r_{p 13}$ and $r_{p 23}$ would lead to a consistency check of the inferred bias factors and increase the signal-to-noise ratio.

As an illustration, we fit the projected 3PCFs measured by Jing \& Börner (2004) for galaxies in the 2dFGRS. Jing \& Börner (2004) have measurements for two samples, one bright sample $\left(M_{b} \leq-19.66\right)$ and one faint sample $\left(-19.66<M_{b} \leq\right.$ 18.5). They also have predictions of reduced projected 3PCFs of the matter for the concordance cosmological model, which are measured in an $N$-body simulation. In their Figures 17 and 18 , they compare the observed $Q_{p, g}$ and the predicted $Q_{p, m}$ for different triangle shapes, which provide the information we need to do the two-parameter fit. A different parameterization is adopted by Jing \& Börner (2004) to characterize a triangle, one parameter $\left(r_{p}\right.$, the length of the shortest side) for the size and two parameters $(u$ and $v)$ for the shape, with the lengths of the three sides being $r_{p}, u r_{p}$, and $(u+v) r_{p}$. In their figures, the 3PCFs are plotted as a function of $v$ (five equal linear bins in the range $0 \leq v \leq 1$ ) for several combinations of $r_{p}$ and $u$. For the two-parameter fit, we choose the case with the largest value of $r_{p}$ they have, i.e., $3.25 h^{-1} \mathrm{Mpc}$ and $u=2.09$. If the $v$ dependence is translated into an angular dependence, the five data points only cover $\Phi$ from $\sim 80^{\circ}$ to $\sim 180^{\circ}$, with the widths of the five angular bins being $12^{\circ}, 13^{\circ}, 15^{\circ}, 19^{\circ}$, and $44^{\circ}$. We see that the last bin smears the angular dependence a lot. So when measuring the $3 \mathrm{PCF}$ s from galaxy clustering data, adopting the $\left(r_{p 13}, r_{p 23}, \Phi\right)$ parameterization and dividing $\Phi$ into narrow bins are probably more suitable to probe the angular dependence than the $\left(r_{p}, u, v\right)$ parameterization. Since the error bars on the measurements are large and the scales are not truly in the weakly nonlinear regime, we cannot obtain robust constraints on galaxy bias parameters. Nevertheless, application of the formalism gives bias factors for galaxies in the bright (faint) sample that are consistent with $b=1.8$ and $b_{2}=0(b=1.1$ and $b_{2}=0$ ), somewhat higher $b$ than expected but not absurdly so.

If the projection is not infinite, the kind of relation shown in equation (16) also holds as long as we use the finite projected correlation functions in the definition of $Q_{p}$ (eq. [18]). Strictly speaking, in this case, the integration of the product of $2 \mathrm{PCF}$ in the right side of equation (17) cannot be written as the product of $w_{p}$ 's except for $\xi\left(r_{p 13}\right) \xi\left(r_{p 23}\right)$. However, the product of $w_{p}$ 's should remain as a good approximation to the results. If one is not satisfied with the approximation, although it is good, one can always compute exact values of these integrations for $Q_{p, m}$ and form $Q_{p, g}$ from the observation in the same way. Based on equation (17), we still have the relation in equation (19).

The result can also be generalized to a projected field, where projected correlations include the effect of the selection function (see Fry \& Thomas 1999). Based on equation (17), it is easy to show that equation (19) holds for $Q_{p}$ defined in terms of selection-function-weighted projected 2PCFs and 3PCFs. Fry \& Thomas (1999) perform a systematic study of projection effects on the reduced 3PCFs. They show that projections that are not deep enough would change the shape of the reduced 3PCF and thus bias the estimation of galaxy bias factors. However, this is based on the comparison with the threedimensional reduced $3 \mathrm{PCF}$ of the matter. Our point here is that once the reduced 3PCF of the matter is calculated by taking account of the selection function, galaxy bias factors can be correctly inferred by comparing it with the observed reduced projected 3PCFs of galaxies. That is, we should always form $Q_{p, g}$ and $Q_{p, m}$ in the same way. The depth of the survey should be much larger than the extent of structures caused by galaxy peculiar velocities so that we can calculate 3PCFs of the matter in real space. Buchalter et al. (2000) also investigate the reduced angular 3PCF. They concentrate on the explicit dependence of the galaxy 3PCF on cosmological parameters, the selection function, and bias, not on the relationship between the reduced $3 \mathrm{PCF}$ of galaxies and that of the matter as done here. Under certain assumptions, their results can be put into the form discussed here. Focusing also on the explicit expression of the galaxy bispectrum, Verde et al. (2000) present a theoretical analysis of the projected bispectrum in spherical harmonics and discuss its application (note that they find that it is not encouraging to constrain bias parameters using projected galaxy catalogs).

\section{SUMMARY AND DISCUSSION}

I investigate the projected $3 \mathrm{PCF}$ and find that it can be put in a simple form if Fourier expansion is introduced. Each Fourier component of the projected $3 \mathrm{PCF}$ is just a transform of the corresponding Fourier component of the bispectrum (eq. [13]). In the weakly nonlinear regime, only the first few Fourier components are significant. The result is parallel to the multipole expansion proposed by Szapudi (2004) for the threedimensional three-point statistics. Fourier expansion reduces the amount of work needed to compute the projected $3 \mathrm{PCF}$ and provides a convenient way to characterize its dependence on the triangle configuration. Projected 2PCFs and 3PCFs formed from redshift-space correlation functions are little affected by redshift-space distortions, which makes it promising to use them to measure galaxy bias factors. In the weakly nonlinear regime, I find that the relation between the reduced threedimensional 3PCFs of galaxies and matter also holds for the reduced projected $3 \mathrm{PCF}$, if these are defined from the projected 3PCF and 2PCF in the same way. The linear bias factor $b$ and the first-order nonlinear bias factor $b_{2}$ thus can be inferred from the reduced projected 3PCFs of galaxies $\left(Q_{p, g}\right)$ formed from redshift-space measurements, through the dependence of $Q_{p, g}$ on the angle between two sides of projected triangles. From the point of view of Fourier expansion, the nonlinear bias 
factor only affects the DC component of $Q_{p}$, and the linear bias factor is given by the ratio of coefficients of other Fourier components of $Q_{p, g}$ and $Q_{p, m}$.

The method of measuring galaxy bias factors from projected 3PCFs can be directly applied to the data from contemporary galaxy redshift surveys (e.g., 2dFGRS and SDSS). The projected 3PCFs of galaxies should be measured on large scales, i.e., in the weakly nonlinear regime, where the bias relation (eq. [19]) predicted by the local bias model applies. The projected 3PCFs of matter can be calculated using the technique introduced in $\S 2$, or they can be measured from cosmological $\mathrm{N}$-body simulations. The inferred bias factors of galaxies would help to constrain cosmological parameters, such as the amplitude of the matter fluctuation power spectrum.

Theoretically, the framework of the halo occupation distribution is often adopted to model galaxy clustering by linking galaxies to dark matter halos (for the three-dimensional 3PCF modeling within this framework, see, e.g., Takada \& Jain 2003; Wang et al. 2004). With the help of this framework, even in the strongly nonlinear regime, galaxy clustering would bring in additional constraining powers on cosmological parameters as well as on the relation between galaxies and matter. In any regime, projected $3 \mathrm{PCF}$, little affected by redshift-space distortions, are useful statistics. On very small scales where the three galaxies of each triplet come from the same dark matter halo, 3PCFs probe the shape of halos and the relative distribution of galaxies and matter inside halos. The projected 3PCF in this regime can be obtained by projecting the real-space 3PCF, which is easy to calculate (Takada \& Jain 2003). On intermediate or large scales where the galaxies of each triplet are from two or three different halos, the calculation of the $3 \mathrm{PCF}$ is simpler in Fourier space than in real space. Fourier expansion would be a useful technique to calculate the projected correlation functions on these scales.

I am grateful to David Weinberg for his encouragement and advice on this work and for his detailed and valuable comments that greatly improved the paper. I thank István Szapudi and Jun Pan for useful suggestions and comments. This work was supported by a Presidential Fellowship from the Graduate School of the Ohio State University and by NSF grant AST 04-07125.
Baugh, C. M., \& Efstathiou, G. 1993, MNRAS, 265, 145

Bernardeau, F. 1992, ApJ, 392, 1

Bernardeau, F., Colombi, S., Gaztañaga, E., \& Scoccimarro, R. 2002, Phys. Rep., 367, 1

Buchalter, A., Kamionkowski, M., \& Jaffe, A. 2000, ApJ, 530, 36

Cole, S., Fisher, K. B., \& Weinberg, D. H. 1994, MNRAS, 267, 785

Colless, M., et al. 2001, MNRAS, 328, 1039

Davis, M., \& Peebles, P. J. E. 1983, ApJ, 267, 465

Efstathiou, G., Bond, J. R., \& White, S. D. M. 1992, MNRAS, 258, P1

Feldman, H. A., Frieman, J. A., Fry, J. N., \& Scoccimarro, R. 2001, Phys. Rev. Lett., 86,1434

Fry, J. N. 1984, ApJ, 279, 499 . 1994, Phys. Rev. Lett., 73, 215

Fry, J. N., \& Gaztañaga, E. 1993, ApJ, 413, 447

Fry, J. N., \& Peebles, P. J. E. 1980, ApJ, 238, 785

Fry, J. N., \& Thomas, D. 1999, ApJ, 524, 591

Goroff, M. H., Grinstein, B., Rey, S.-J., \& Wise, M. 1986, ApJ, 311, 6

Jain, B., \& Bertschinger, E. 1994, ApJ, 431, 495

Jing, Y. P., \& Börner, G. 1998, ApJ, 503, 37

Jing, Y. P., Mo, H. J., \& Börner, G. 1991, A\&A, 252, 449

2004, ApJ, 607, 140

Juszkiewicz, R., Weinberg, D. H., Amsterdamski, P., Chodorowski, M., \& Bouchet, F. 1995, ApJ, 442, 39

\section{REFERENCES}

Kamionkowski, M., \& Buchalter, A. 1999, ApJ, 514, 7

Kayo, I., et al. 2004, PASJ, 56, 415

Landy, S. A., \& Szalay, A. S. 1993, ApJ, 412, 64

Matsubara, T. 1995, Prog. Theor. Phys., 94, 1151

Peebles, P. J. E. 1980, The Large-Scale Structure of the Universe (Princeton: Princeton Univ. Press)

Peebles, P. J. E., \& Groth, E. J. 1975, ApJ, 196, 1

Scoccimarro, R., Colombi, S., Fry, J. N., Frieman, J. A., Hivon, E., \& Melott, A. 1998, ApJ, 496, 586

Scoccimarro, R., Feldman, H. A., Fry, J. N., \& Frieman, J. A. 2001, ApJ, 546,652

Scoccimarro, R., \& Frieman, J. 1996, ApJS, 105, 37

1999, ApJ, 520, 35

Szapudi, I. 2004, ApJ, 605, L89

Takada, M., \& Jain, B. 2003, MNRAS, 340, 580

Verde, L., Heavens, A. F., \& Matarrese, S. 2000, MNRAS, 318, 584

Verde, L., et al. 2002, MNRAS, 335, 432

Wang, Y., Yang, X. H., Mo, H. J., van den Bosch, F. C., \& Chu, Y. C. 2004, MNRAS, 353, 287

York, D., et al. 2000, AJ, 120, 1579 